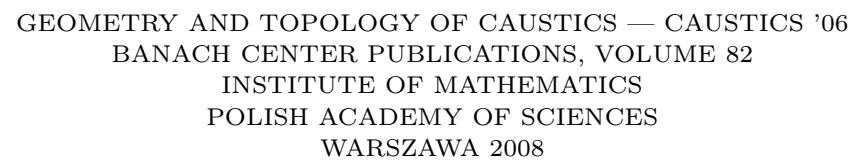

\title{
BIFURCATIONS IN SYMPLECTIC SPACE
}

\author{
G. ISHIKAWA \\ Department of Mathematics, Hokkaido University \\ Sapporo 060-0810, Japan \\ E-mail: ishikawa@math.sci.hokudai.ac.jp \\ S. JANECZKO \\ Institute of Mathematics, Polish Academy of Sciences \\ Sniadeckich 8, 00-956 Warszawa, Poland \\ and \\ Faculty of Mathematics and Information Science, Warsaw University of Technology \\ Pl. Politechniki 1, 00-661 Warszawa, Poland \\ E-mail: Janeczko@alpha.mini.pw.edu.pl
}

\begin{abstract}
In this paper we take new steps in the theory of symplectic and isotropic bifurcations, by solving the classification problem under a natural equivalence in several typical cases. Moreover we define the notion of coisotropic varieties and formulate also the coisotropic bifurcation problem. We consider several symplectic invariants of isotropic and coisotropic varieties, providing illustrative examples in the simplest non-trivial cases.
\end{abstract}

1. Introduction. Let $H: M^{2 n} \rightarrow \mathbf{R}^{n-k}, 0 \leq k \leq n-1$, be a coisotropic fibration of the symplectic space $\left(M^{2 n}, \omega\right), n \geq 2$. We define $H$ as a momentum map, $H=$ $\left(H_{1}, \ldots, H_{n-k}\right): M^{2 n} \rightarrow \mathbf{R}^{n-k}$ with $\left\{H_{i}\right\}$ in involution, i.e. $\left\{H_{i}, H_{j}\right\} \equiv 0$ for all $1 \leq$ $i, j \leq n-k$, where $\left\{H_{i}, H_{j}\right\}$ is defined by the equality $\left\{H_{i}, H_{j}\right\} \omega^{n}=d H_{i} \wedge d H_{j} \wedge \omega^{n-1}$.

If $\bar{q} \in \mathbf{R}^{n-k}$ is a regular value of $H$ then $H^{-1}(\bar{q})$ is a coisotropic submanifold of $\left(M^{2 n}, \omega\right)$. By the Jacobi-Liouville theorem, locally there exist relative Darboux coordinates $p_{1}, \ldots, p_{n}, q_{1}, \ldots, q_{n}$ on $M^{2 n}$ such that $\omega=\sum_{i=1}^{n} d p_{i} \wedge d q_{i}$ and $H(p, q)=(\bar{q})=$ $\left(q_{k+1}, \ldots, q_{n}\right)$. To each coisotropic submanifold $H^{-1}(\bar{q})$, or equivalently to each $\bar{q} \in \mathbf{R}^{n-k}$

2000 Mathematics Subject Classification: 70K50, 58K05, 58K60, $13 \mathrm{C} 14$.

Key words and phrases: symplectic manifold, Lagrangian variety, liftable equivalence, coisotropic variety, bifurcations of isotropic varieties.

The work was supported by EC FP6 MC ToK programmes TODEQ, MTKD-CT-2005030042 and SPADE2, MTKD-CT-2004-014508.

The paper is in final form and no version of it will be published elsewhere. 
we assign the symplectic space, i.e. the reduced symplectic space $M_{\bar{q}}$ given by the canonical reduction (Marsden-Weinstein reduction [19]) $\pi_{\bar{q}}$;

$$
\bar{q} \mapsto \pi_{\bar{q}}: H^{-1}(\bar{q}) \rightarrow H^{-1}(\bar{q}) / \sim_{\bar{q}} \equiv M_{\bar{q}}
$$

along each fiber $H^{-1}(\bar{q})$ endowed with the symplectic structure $\mu_{\bar{q}}$ uniquely defined by the reduction formula

$$
\pi_{\bar{q}}^{*} \mu_{\bar{q}}=\left.\omega\right|_{H^{-1}(\bar{q})} .
$$

In Darboux coordinates we assume

$$
\pi_{\bar{q}}\left(p_{1}, \ldots, p_{k}, q_{1}, \ldots, q_{k}, p_{k+1}, \ldots, p_{n}\right)=\left(p_{1}, \ldots, p_{k}, q_{1}, \ldots, q_{k}\right)
$$

and identify $\left(M_{\bar{q}}, \mu_{\bar{q}}\right)$ with $T^{*} \mathbf{R}^{k}$ endowed with its canonical Liouville form.

Collecting all the reduction projections we get the total projection

$$
\begin{gathered}
\pi: M^{2 n} \rightarrow T^{*} \mathbf{R}^{k} \times \mathbf{R}^{n-k}=: N^{n+k}, \\
\pi(p, q)=\left(p_{1}, \ldots, p_{k}, q_{1}, \ldots, q_{k}, q_{k+1}, \ldots, q_{n}\right)
\end{gathered}
$$

such that the following diagram commutes:

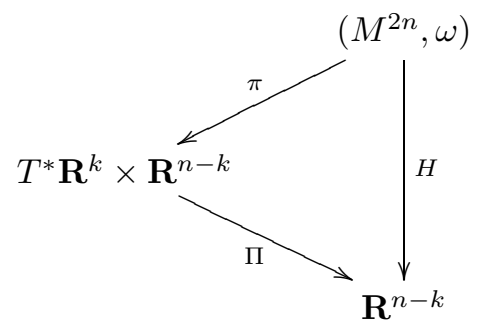

Definition 1.1. Any map germ $F:\left(\mathbf{R}^{m}, 0\right) \rightarrow T^{*} \mathbf{R}^{k} \times \mathbf{R}^{n-k}, n-k \leq m<n+k$, is called a bifurcation in symplectic space $T^{*} \mathbf{R}^{k}$ (or briefly a symplectic bifurcation).

The bifurcation parameters now are regular values $(\bar{q})$ of the momentum map $H$. A bifurcating family of varieties in $T^{*} \mathbf{R}^{k}$ is defined from the above diagram as

$$
\mathbf{R}^{n-k} \ni \bar{q} \mapsto F\left(\mathbf{R}^{m}\right) \cap \Pi^{-1}(\bar{q}) \subset T^{*} \mathbf{R}^{k} \times\{\bar{q}\} .
$$

Let $F:\left(\mathbf{R}^{m}, 0\right) \rightarrow T^{*} \mathbf{R}^{k} \times \mathbf{R}^{n-k}$ be a symplectic bifurcation. We call it an isotropic bifurcation if and only if $F$ lifts to a smooth map-germ $\tilde{F}:\left(\mathbf{R}^{m}, 0\right) \rightarrow\left(M^{2 n}, \omega\right)$ which is isotropic, i.e. $\tilde{F}^{*} \omega=0$ and the following diagram commutes:

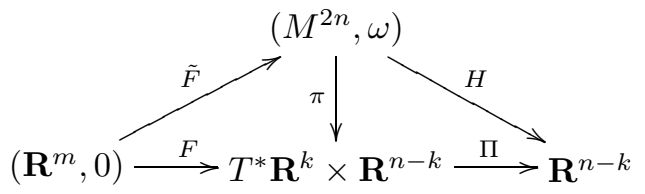

So $F=\pi \circ \tilde{F}$, and $\tilde{F}$ is called an isotropic (coisotropic) lifting of $F$.

By $L=\tilde{F}\left(\mathbf{R}^{m}\right)$ we denote the parametric isotropic variety (Lagrangian if $m=n$ ). Then $\pi(L)$ is a family of $(m-n+k)$-dimensional isotropic varieties in $T^{*} \mathbf{R}^{k}$ parametrized by $\mathbf{R}^{n-k}$. If $m=n$ then this is a family of Lagrangian varieties in $T^{*} \mathbf{R}^{k}$. Now we have the bifurcating family

$$
\mathbf{R}^{n-k} \ni \bar{q} \mapsto \pi\left(H^{-1}(\bar{q}) \cap L\right) \subset T^{*} \mathbf{R}^{k} \times\{\bar{q}\} .
$$


In $\S 2$, we give a natural equivalence relation, liftable equivalence, for symplectic bifurcations. In $\S 3$, we study the case $m=n-k$, of symplectic bifurcations of finite sets. In particular, the generic classification is provided for the two-parameter isotropic bifurcations of corank one $(n-k=2)$.

The case $m=n-k+1$, of bifurcation of curves in symplectic space, was treated in [8]. In particular, in the case $m=n-k+1, k=1$, of symplectic bifurcations of planar curves, the classification problem has been solved in a satisfactory manner [8], [10].

A few results are known on symplectic bifurcations of surfaces $(m=n-k+2)$. In $\S 4$ we provide a result on one-parameter symplectic bifurcations of Lagrange varieties in $T^{*} \mathbf{R}^{2}(n=3, k=2, m=3)$.

In $\S 5$, we introduce the notion of coisotropic varieties and coisotropic bifurcations. Let $F:\left(\mathbf{R}^{m}, 0\right) \rightarrow T^{*} \mathbf{R}^{k} \times \mathbf{R}^{n-k}$ be a symplectic bifurcation. Suppose that $m \geq n$. We call $F$ a coisotropic bifurcation if $F$ lifts to a smooth map-germ $\tilde{F}:\left(\mathbf{R}^{m}, 0\right) \rightarrow\left(M^{2 n}, \omega\right)$ which is "coisotropic". Here, in general, we call a map-germ $f:\left(\mathbf{R}^{m}, 0\right) \rightarrow T^{*} \mathbf{R}^{k}$ coisotropic if $f$ lifts to an isotropic map-germ $\tilde{f}:\left(\mathbf{R}^{m}, 0\right) \rightarrow T^{*} \mathbf{R}^{m}=T^{*} \mathbf{R}^{k} \times T^{*} \mathbf{R}^{m-k}$ characterized via the symplectic projection $T^{*} \mathbf{R}^{k} \times T^{*} \mathbf{R}^{m-k} \rightarrow T^{*} \mathbf{R}^{k}$. We give a typical example of coisotropic map-germs and coisotropic bifurcations.

$\S 6$ is devoted to invariants for coisotropic varieties, analogous to those for isotropic varieties.

In the last section $(\S 7)$ we observe that coisotropic varieties are obtained from isotropic (or Lagrangian) bifurcations as loci (unions) of Lagrangian varieties depending on the bifurcation parameter and coisotropic varieties are characterised by this property under a certain condition.

2. Liftable equivalence. Let $F_{1}, F_{2}:\left(\mathbf{R}^{m}, 0\right) \rightarrow\left(T^{*} \mathbf{R}^{n} \times \mathbf{R}^{n-k}, 0\right)=:(N, 0)$ be two bifurcation germs. We say that $F_{1}, F_{2}$ are liftably equivalent (see [8]) if there are diffeomorphism-germs $\psi, \phi$ and a symplectomorphism-germ $\Phi$ such that the following diagram commutes:

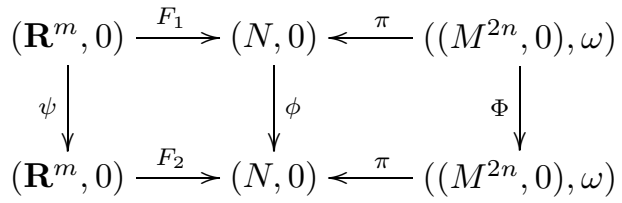

The diffeomorphism-germ $\phi$ in such a diagram is called symplectically liftable. Thus we consider the classification of bifurcation-germ singularities according to the group $\mathcal{G}_{\text {symp }}$ of symplectically liftable diffeomorphisms of $(N, 0)$ which is a subgroup of the group $\mathcal{G}$ of diffeomorphism-germs. The group $\mathcal{G}_{\text {symp }}$ was explicitly described in [8].

Proposition 2.1 ([8]). A diffeomorphism-germ $\phi:(N, 0) \rightarrow(N, 0)$ is symplectically liftable if and only if $\phi$ is a family of symplectic diffeomorphisms of $T^{*} \mathbf{R}^{k}$ parametrized by $\bar{q}=\left(q_{k+1}, \ldots, q_{n}\right)$. Moreover $\phi$ has a symplectic lifting $\Phi:\left(\left(M^{2 n}, 0\right), \omega\right) \rightarrow\left(\left(M^{2 n}, 0\right), \omega\right)$ preserving fibers of $H$, i.e. there exists a diffeomorphism-germ $\sigma:\left(\mathbf{R}^{n-k}, 0\right) \rightarrow\left(\mathbf{R}^{n-k}, 0\right)$ such that the following diagram commutes: 


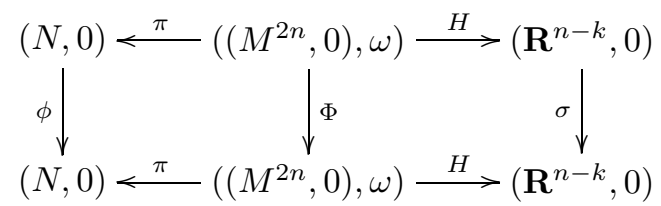

Any vector field $X$ over $N$ generating a liftable equivalence is given by

$$
X\left(q_{1}, p_{1}, \ldots, q_{k}, p_{k} ; \bar{q}\right)=X_{h_{\bar{q}}}\left(q_{1}, p_{1}, \ldots, q_{k}, p_{k}\right)+\sum_{i=1}^{n-k} a_{i}(\bar{q}) \frac{\partial}{\partial q_{k+i}}
$$

for some functions $a_{i}(\bar{q}), i=1, \ldots, n-k$, and the Hamiltonian function $h_{\bar{q}}\left(q_{1}, p_{1}, \ldots, q_{k}, p_{k}\right)$ $=h\left(q_{1}, p_{1}, \ldots, q_{k}, p_{k} ; \bar{q}\right)$ of the Hamiltonian vector-field $X_{h_{\bar{q}}}$ over $T^{*} \mathbf{R}^{k}$ for each $\bar{q} \in$ $\mathbf{R}^{n-k}$. We easily see that the lifted Hamiltonian vector-field $\tilde{X}$ over $\left(M^{2 n}, \omega\right)$ generating the lifted symplectic equivalence is defined by the Hamiltonian

$$
\tilde{h}(p, q)=h\left(q_{1}, p_{1}, \ldots, q_{k}, p_{k} ; \bar{q}\right)+\sum_{i=1}^{n-k} p_{k+i} a_{i}(\bar{q}) .
$$

Let $\tilde{F}_{1}, \tilde{F}_{2}:\left(\mathbf{R}^{m}, 0\right) \rightarrow\left(M^{2 n}, 0\right)$ be two map-germs into a symplectic space $\left(M^{2 n}, \omega\right)$. Definition 2.1. $\tilde{F}_{1}, \tilde{F}_{2}$ are called $H$-symplectically equivalent if there exist a symplectomorphism $\Phi:\left(\left(M^{2 n}, 0\right), \omega\right) \rightarrow\left(\left(M^{2 n}, 0\right), \omega\right)$ and diffeomorphisms $\psi:\left(\mathbf{R}^{m}, 0\right) \rightarrow\left(\mathbf{R}^{m}, 0\right)$, $\sigma:\left(\mathbf{R}^{n-k}, 0\right) \rightarrow\left(\mathbf{R}^{n-k}, 0\right)$ such that the following diagram commutes:

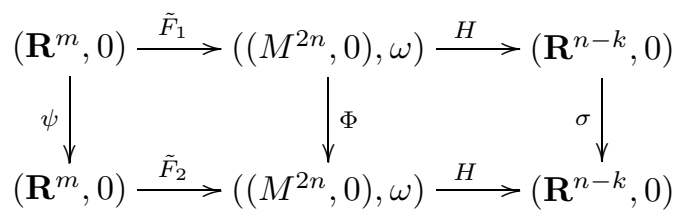

Now we have immediately

Corollary 2.1. If $F_{1}, F_{2}:\left(\mathbf{R}^{m}, 0\right) \rightarrow\left(T^{*} \mathbf{R}^{n} \times \mathbf{R}^{n-k}, 0\right)$ are liftably equivalent and have isotropic liftings, then their isotropic liftings $\tilde{F}_{1}, \tilde{F}_{2}:\left(\mathbf{R}^{m}, 0\right) \rightarrow\left(M^{2 n}, 0\right)$ are $H$ symplectically equivalent.

3. Bifurcations of finite sets of points. If $m=n-k$ then any map-germ $F$ : $\left(\mathbf{R}^{n-k}, 0\right) \rightarrow T^{*} \mathbf{R}^{k} \times \mathbf{R}^{n-k}$ will be called a finite point set symplectic bifurcation in $\left(T^{*} \mathbf{R}^{k}, \omega_{k}\right)$ provided $f=\Pi \circ F$ is a finite-to-one map-germ. In this case the symplectic bifurcations are classified by singularities of map-germs $f:\left(\mathbf{R}^{n-k}, 0\right) \rightarrow\left(\mathbf{R}^{n-k}, 0\right)$. In local coordinates

$$
f\left(x_{1}, \ldots, x_{n-k}\right)=\left(q_{k+1}(x), \ldots, q_{n}(x)\right)
$$

and we have a bifurcating family of collections of points

$$
\bar{q} \mapsto\left\{F\left(\left\{f^{-1}(\bar{q})\right\}\right)\right\}
$$

all staying in the image of $F$ projected into $T^{*} \mathbf{R}^{k}$. It will be an isotropic bifurcation if $F$ lifts to an isotropic map-germ $\tilde{F}:\left(\mathbf{R}^{n-k}, 0\right) \rightarrow\left(\mathbf{R}^{2 n}, \omega\right), \tilde{F}^{*} \omega=0$. 
If $n-k=1$ then all one-parameter symplectic bifurcations of finite sets of points in $T^{*} \mathbf{R}^{k}$ are obviously isotropic and classified by critical values of a smooth function $f(x)=q_{k+1}(x)$.

Singularities of $F$ are classified by the symplectic singularities of divergent mapping diagrams

$$
T^{*} \mathbf{R}^{k} \stackrel{\rho}{\longleftarrow} \mathbf{R}^{n-k} \stackrel{f}{\longrightarrow} \mathbf{R}^{n-k},
$$

where $F=(\rho, f)$. They are initially classified by singularities of mappings $f$. It is natural to ask if generic singularities of map-germs $F$ can be lifted to isotropic map-germs $\tilde{F}$.

For the case $n-k=2$ we have

Proposition 3.1. All smooth map-germs $F:\left(\mathbf{R}^{2}, 0\right) \rightarrow\left(T^{*} \mathbf{R}^{k} \times \mathbf{R}^{2}, 0\right)$ such that $f:$ $\left(\mathbf{R}^{2}, 0\right) \rightarrow\left(\mathbf{R}^{2}, 0\right)$ is a singularity of corank one are regular isotropic bifurcations, i.e. $F$ is liftable to an immersive isotropic map-germ $\tilde{F}:\left(\mathbf{R}^{2}, 0\right) \rightarrow\left(\mathbf{R}^{2(k+2)}, \omega\right), \tilde{F}^{*} \omega=0$.

Proof. In Darboux coordinates we have

$$
F(x)=(\rho(x), f(x))=\left(\bar{p}(x), \bar{q}(x) ; q_{k+1}(x), q_{k+2}(x)\right),
$$

where $\bar{q}=\left(q_{1}, \ldots, q_{k}\right), \bar{p}=\left(p_{1}, \ldots, p_{k}\right)$,

$$
\omega=\omega_{k}+d p_{k+1} \wedge d q_{k+1}+d p_{k+2} \wedge d q_{k+2}, \quad \omega_{k}=\sum_{i=1}^{k} d p_{i} \wedge d q_{i} .
$$

An isotropic lifting $\tilde{F}$ is given by a solution of the equation $\tilde{F}^{*} \omega=0$ such that $\pi \circ \tilde{F}=F$. We write

$$
\rho^{*} \omega_{k}+\tilde{f}^{*}\left(d p_{k+1} \wedge d q_{k+1}+d p_{k+2} \wedge d q_{k+2}\right)=0,
$$

where $\tilde{f}(x)=\left(p_{k+1}(x), p_{k+2}(x), f(x)\right)$ and $p_{k+1}(x), p_{k+2}(x)$ are smooth function-germs on $\left(\mathbf{R}^{2}, 0\right)$.

Since $f$ is a map-germ of corank one, by right equivalence we reduce it to the form $f(x)=\left(x_{1}, q_{k+2}(x)\right)$ and from equation (1) we have

$$
\frac{\partial p_{k+1}}{\partial x_{2}}(x)=g(x)-\left(\frac{\partial q_{k+2}}{\partial x_{1}}(x) \frac{\partial p_{k+2}}{\partial x_{2}}(x)-\frac{\partial q_{k+2}}{\partial x_{2}}(x) \frac{\partial p_{k+2}}{\partial x_{1}}(x)\right) .
$$

Taking $p_{k+2}(x)$ such that $\frac{\partial p_{k+2}}{\partial x_{2}}(0) \neq 0$ we may represent $p_{k+1}(x)$ as the integral

$$
p_{k+1}(x)=\int\left(g(x)-\left(\frac{\partial q_{k+2}}{\partial x_{1}}(x) \frac{\partial p_{k+2}}{\partial x_{2}}(x)-\frac{\partial q_{k+2}}{\partial x_{2}}(x) \frac{\partial p_{k+2}}{\partial x_{1}}(x)\right)\right) d x_{2} .
$$

Proposition 3.2. Normal forms of corank one generic isotropic bifurcations $F=(\rho, f)$ : $\left(\mathbf{R}^{2}, 0\right) \rightarrow\left(T^{*} \mathbf{R} \times \mathbf{R}^{2}, 0\right)$ of fold and cusp type are liftable equivalent to

1. (fold) $f(x)=\left(x_{1}, x_{2}^{2}\right)$ :

$$
\rho(x)=\left(x_{1} \phi\left(x_{1}, x_{2}\right), x_{1}+x_{2}\right) ;
$$

2. (cusp) $f(x)=\left(x_{1}, x_{2} x_{1}+x_{2}^{3}\right)$ :

$$
\rho(x)=\left(x_{1} \phi_{1}\left(x_{1}, x_{2}\right), x_{2}+\phi_{2}\left(x_{1}, x_{1} x_{2}+x_{2}^{3}\right) .\right.
$$

Proof. We prove the representative case of "fold". By right-left equivalence of $f$ we write

$$
\rho\left(x_{1}, x_{2}\right) \rightarrow\left(P_{1}\left(x_{1}, x_{2}\right), Q_{1}\left(x_{1}, x_{2}\right)\right), \quad f\left(x_{1}, x_{2}\right)=\left(x_{1}, x_{2}^{2}\right) .
$$


The group of lowerable diffeomorphism-germs acting on the source space of $f$ consists of diffeomorphisms of the form

$$
\left(x_{1}, x_{2}\right) \rightarrow\left(\alpha\left(x_{1}, x_{2}^{2}\right), x_{2} \beta\left(x_{1}, x_{2}^{2}\right)\right) .
$$

Let us write $Q_{1}$ in the form

$$
Q_{1}\left(x_{1}, x_{2}\right)=u\left(x_{1}, x_{2}^{2}\right)+x_{2} w\left(x_{1}, x_{2}^{2}\right)
$$

with a generic assumption

$$
\frac{\partial u}{\partial x_{1}}(0) \neq 0, \quad w(0) \neq 0 .
$$

Then by the lowerable diffeomorphism

$$
\left(x_{1}, x_{2}\right) \rightarrow\left(X_{1}\left(x_{1}, x_{2}\right), X_{2}\left(x_{1}, x_{2}\right)\right)=\left(u\left(x_{1}, x_{2}^{2}\right), x_{2} w\left(x_{1}, x_{2}^{2}\right)\right)
$$

we get the normal form

$$
Q_{1}\left(x_{1}, x_{2}\right)=x_{1}+x_{2} .
$$

Using the symplectomorphism of $T^{*} \mathbf{R}^{1}$ preserving this normal form we reduce $P_{1}$ to $P_{1}\left(x_{1}, x_{2}\right)=x_{1} \phi\left(x_{1}, x_{2}\right)$ for some smooth function $\phi$. The proof of the "cusp" case is analogous, using the lowerable vector fields on the source space of the map-germ with cusp singularity.

4. Symplectic bifurcations of Lagrangian surfaces. Let $f:\left(\mathbf{R}^{m}, 0\right) \rightarrow T^{*} \mathbf{R}^{n}$ be an isotropic map-germ $\left(f^{*} \omega=0\right)$. Recall that, if $m=1$, then any map-germ $f$ is isotropic. We consider isotropic surfaces and their symplectic bifurcations. First we recall the basic terminology:

Definition 4.1. Two map-germs $f, f^{\prime}:\left(\mathbf{R}^{m}, 0\right) \rightarrow\left(\mathbf{R}^{2 n}, \omega\right)$ are called symplectomorphic (resp. diffeomorphic) if $f^{\prime}$ is transformed to $f$ by a symplectomorphism (resp. diffeomorphism) on $\mathbf{R}^{2 n}$ up to re-parametrization on $\mathbf{R}^{m}$.

Note that the term "diffeomorphic" is a replacement of "right-left equivalent". Then we have:

Proposition 4.1 ([6]). Let $f:\left(\mathbf{R}^{2}, 0\right) \rightarrow T^{*} \mathbf{R}^{2}$ be isotropic. Suppose that $f$ is diffeomorphic to $f_{\text {ou }}(t, u)=\left(u t, t^{2}, \frac{2}{3} t^{3}, u\right)=\left(p_{1}, q_{1}, p_{2}, q_{2}\right)$. Then $f$ is symplectomorphic to $f_{\text {ou }}$ (Darboux-type theorem). Moreover, for any $n$ there exists a class of open umbrellas characterised by the symplectic structural stability, and for them, the Darboux type theorem holds.

For submanifolds of a symplectic space, the symplectic classification is reduced to the diffeomorphic classification of restrictions of the symplectic form to submanifolds (Darboux-Givental theorem [3]). We refer to a generalisation of the Darboux-Givental theorem to the singular case.

Proposition 4.2 (Domitrz, Janeczko, Zhitomirskii, [4]). For any $N, N^{\prime} \subset \mathbf{R}^{2 n}$ quasihomogeneous, for any symplectic forms $\omega, \omega^{\prime}$ on $\mathbf{R}^{2 n},(N, \omega)$ and $\left(N^{\prime}, \omega^{\prime}\right)$ are symplectomorphic if and only if the algebraic restrictions $[\omega]_{N}$ and $\left[\omega^{\prime}\right]_{N^{\prime}}$ are diffeomorphic.

Thus we have: 
COROLlary 4.1. Algebraic restrictions of symplectic forms to an open umbrella are diffeomorphic to each other.

There is the notion of symplectic codimension sp-codim $(f)$ for an isotropic map-germ $f$. The number sp-codim $(f)$ is characterised as the minimal number of parameters of a symplectically versal unfolding of $f([9])$.

Proposition $4.3([9])$. sp-codim $(f)$ is a diffeomorphism invariant for isotropic normalisations $f:\left(\mathbf{R}^{n}, 0\right) \rightarrow T^{*} \mathbf{R}^{n}$. If isotropic map-germs $f$ and $f^{\prime}$ are diffeomorphic, then $\operatorname{sp}-\operatorname{codim}(f)=\operatorname{sp}-\operatorname{codim}\left(f^{\prime}\right)$.

In the complex analytic case, if $\operatorname{codim} \Sigma(f) \geq 2$, then

where

$$
\operatorname{sp}-\operatorname{codim}(f)=\operatorname{dim}_{\mathbb{C}} \mathcal{R}_{f} / f^{*} \mathcal{O}_{2 n},
$$

$$
\mathcal{R}_{f}:=\left\{h \in \mathcal{O}_{n} \mid d h \in \mathcal{O}_{n} \cdot d f\right\} .
$$

In the case $n=1$, we have

$$
\operatorname{sp-\operatorname {codim}}(f)=\operatorname{dim}_{\mathbb{C}} \mathcal{O}_{1} / f^{*} \mathcal{O}_{2} .
$$

Moreover the difference of differential/symplectic classification is given by

$$
\operatorname{gh}(f):=\operatorname{dim}_{\mathbb{C}} \mathcal{G}_{f} / f^{*} \mathcal{O}_{2 n},
$$

the symplectic defect or ghost number, where

$$
\mathcal{G}_{f}:=\left\{h \in \mathcal{O}_{n} \mid d h \in f^{*} \Omega_{2 n}^{1}\right\}=\left\{h \in \mathcal{O}_{n} \mid d h \in f^{*} \mathcal{O}_{2 n} \cdot d f\right\} .
$$

Note that

$$
\mathcal{R}_{f} \supseteq \mathcal{G}_{f} \supseteq f^{*} \mathcal{O}_{2 n}, \quad f^{*}: \mathcal{O}_{n} \leftarrow \mathcal{O}_{2 n}
$$

EXAmple 4.1. Recall the open umbrella $f_{\text {ou }}=\left(u t, t^{2}, \frac{2}{3} t^{3}, u\right):\left(\mathbf{R}^{2}, 0\right) \rightarrow\left(\mathbf{R}^{4}, 0\right)$. Then we have $d h(t, u) \in\left\langle d(u t), d\left(t^{2}\right), d\left(\frac{2}{3} t^{3}\right), d u\right\rangle_{\mathcal{O}_{2}}=\langle t d t, u d t, d u\rangle_{\mathcal{O}_{2}}$. This condition is equivalent to $h=a\left(t^{2}, t^{3}, u t, u\right)$ for some smooth function $a$. Therefore we have $\mathcal{R}_{f}=\mathcal{G}_{f}=f^{*} \mathcal{O}_{4}$, thus sp-codim $(f)=0$ in this case.

As examples of unstable isotropic mappings, we refer to:

EXAMPLE 4.2. The multiple open umbrella $f_{\text {mou }}^{ \pm}:\left(\mathbf{R}^{2}, 0\right) \rightarrow T^{*} \mathbf{R}^{2}$, where

$$
f_{\text {mou }}^{ \pm}(t, u)=\left(t^{3} \pm u^{2} t, t^{2}, \pm \frac{4}{3} u t^{3}, u\right),
$$

is an isotropic map-germ of corank $\leq 1$ with $\operatorname{sp-codim}(f)=1$. $f_{\text {mou }}^{+}$is not symplectomorphic to $f_{\text {mou }}^{-}$. In fact $f_{\text {mou }}^{+}$and $f_{\text {mou }}^{-}$are not diffeomorphic.

For the multiple open umbrella, $\mathcal{R}_{f} \supsetneq \mathcal{G}_{f}=f^{*} \mathcal{O}_{2 n}$ : There is no ghost in this case.

Now we define the symplectic bifurcation $F:\left(\mathbf{R}^{3}, 0\right) \rightarrow T^{*} \mathbf{R}^{2} \times \mathbf{R}$ by

$$
F(t, u, v)=\left(t^{2}, u, t^{3} \pm\left(u^{2}+v\right) t, \pm \frac{4}{3} u t^{3} ; v\right) .
$$

The symplectic bifurcation $F$ lifts to $\tilde{F}:\left(\mathbf{R}^{3}, 0\right) \rightarrow T^{*} \mathbf{R}^{3}$,

$$
\tilde{F}(t, u, v)=\left(t^{3} \pm\left(u^{2}+v\right) t, t^{2}, \pm \frac{4}{3} u t^{3}, u ; \pm \frac{2}{3} t^{3}, v\right)=\left(p_{1}, q_{1}, p_{2}, q_{2} ; p_{3}, q_{3}\right)
$$

which is isotropic. Therefore $F$ is an isotropic bifurcation. 
EXAmple 4.3. Let $f_{\lambda}(u, t):=\left(t^{5}+u t^{3}+\lambda u^{2} t, t^{2}, \frac{2}{5} t^{5}+\frac{4}{3} \lambda u t^{3}, u\right)=\left(p_{1}, q_{1}, p_{2}, q_{2}\right), \lambda \neq$ $0, \frac{21}{100}$. Then the family $f_{\lambda}$ of isotropic map-germs is trivialised by diffeomorphisms, but $\lambda$ gives the "symplectic moduli". We have sp-codim $\left(f_{\lambda}\right)=2$, and $\mathcal{R}_{f_{\lambda}} \supsetneq \mathcal{G}_{f_{\lambda}} \supsetneq f_{\lambda}{ }^{*} \mathcal{O}_{2 n}$.

5. Coisotropic varieties and bifurcations. Let $\left(\mathbf{R}^{2 n}, \omega\right)$ and $\left(\mathbf{R}^{2 k}, \mu\right)$ be two symplectic spaces (with Darboux forms $\omega$ and $\mu$ ). Actually $\omega=\sum_{i=1}^{n} d p_{i} \wedge d q_{i}$ for the coordinates $\left(p_{1}, \ldots, p_{n}, q_{1}, \ldots, q_{n}\right)$ of $\mathbf{R}^{2 n}$. Similarly $\mu=\sum_{i=k+1}^{n+k} d p_{i} \wedge d q_{i}$ for the coordinates $\left(p_{n+1}, \ldots, p_{k+n}, q_{n+1}, \ldots, q_{k+n}\right)$ of $\mathbf{R}^{2 k}$. Suppose $0<k<n$.

Definition 5.1. A smooth map-germ $f:\left(\mathbf{R}^{n+k}, 0\right) \rightarrow\left(\mathbf{R}^{2 n}, \omega\right)$ is called coisotropic if there exists a smooth map germ $g:\left(\mathbf{R}^{n+k}, 0\right) \rightarrow\left(\mathbf{R}^{2 k}, \mu\right)$ such that $f^{*} \omega=g^{*} \mu$. Moreover, if we can choose $g$ to be of maximal rank, then we call $f$ a coisotropic map-germ with regular reduction.

We can interpret the condition $f^{*} \omega=g^{*} \mu$ as the condition that the map-germ $\tilde{f}=$ $(f, g):\left(\mathbf{R}^{n+k}, 0\right) \rightarrow\left(\mathbf{R}^{2 n} \times \mathbf{R}^{2 k}, \omega \ominus \mu\right)$ is isotropic, i.e.

$$
\tilde{f}^{*}(\omega \ominus \mu)=0,
$$

where $\omega \ominus \mu=\pi_{1}^{*} \omega-\pi_{2}^{*} \mu$, for the projections $\pi_{1}: \mathbf{R}^{2(n+k)}=\mathbf{R}^{2 n} \times \mathbf{R}^{2 k} \rightarrow \mathbf{R}^{2 n}$ and $\pi_{2}: \mathbf{R}^{2(n+k)}=\mathbf{R}^{2 n} \times \mathbf{R}^{2 k} \rightarrow \mathbf{R}^{2 k}$. Thus a coisotropic map-germ $f$ is the image of a parametric Lagrangian variety $\tilde{f}$ under the symplectic projection $\pi_{1}$. Then we call $\tilde{f}$ a Lagrangian lifting of the coisotropic map-germ $f$.

Then naturally we introduce the following notion:

Definition 5.2. Let $F:\left(\mathbf{R}^{m}, 0\right) \rightarrow T^{*} \mathbf{R}^{k} \times \mathbf{R}^{n-k}$ be a symplectic bifurcation. We call $F$ a coisotropic bifurcation if there exists a coisotropic map-germ $\tilde{F}:\left(\mathbf{R}^{m}, 0\right) \rightarrow T^{*} \mathbf{R}^{n}=$ $T^{*} \mathbf{R}^{k} \times T^{*} \mathbf{R}^{n-k}$ such that $\pi \circ \tilde{F}=F$. Here $\pi: T^{*} \mathbf{R}^{k} \times T^{*} \mathbf{R}^{n-k} \rightarrow T^{*} \mathbf{R}^{k} \times \mathbf{R}^{n-k}$ is the product of the identity of $T^{*} \mathbf{R}^{k}$ and the canonical bundle projection $T^{*} \mathbf{R}^{n-k} \rightarrow \mathbf{R}^{n-k}$.

Conversely suppose $f:\left(\mathbf{R}^{m}, 0\right) \rightarrow T^{*} \mathbf{R}^{n}=T^{*} \mathbf{R}^{k} \times T^{*} \mathbf{R}^{n-k}$ is a coisotropic mapgerm. Then $\pi \circ f$ is a coisotropic bifurcation. Note that we can choose any Lagrangian projection $\pi: T^{*} \mathbf{R}^{n-k}=\mathbf{R}^{2(n-k)} \rightarrow \mathbf{R}^{n-k}$ to produce a coisotropic projection $H$ : $T^{*} \mathbf{R}^{n} \rightarrow \mathbf{R}^{n-k}$, provided $\pi \circ f$ is a submersion. The liftable equivalence of bifurcations is finer than the symplectic equivalence.

Now we study the coisotropic varieties, in particular, those with regular reduction.

LEMMA 5.1. Let $g$ and $g^{\prime}:\left(\mathbf{R}^{n+k}, 0\right) \rightarrow \mathbf{R}^{2 k}$ be submersion-germs satisfying $g^{*} \mu=g^{*} \mu$. Then there exists a unique symplectomorphism $\varphi:\left(\mathbf{R}^{2 k}, g(0)\right) \rightarrow\left(\mathbf{R}^{2 k}, g^{\prime}(0)\right)$ such that $g^{\prime}=\varphi \circ g$.

Proof. The fibers of $g$ are characterized as the characteristic foliation defined by the distribution

$$
D:=\left\{v \in T \mathbf{R}^{n+k} \mid i_{v}\left(g^{*} \mu\right)=0\right\} .
$$

Thus there exists a unique diffeomorphism $\varphi:\left(\mathbf{R}^{2 k}, g(0)\right) \rightarrow\left(\mathbf{R}^{2 k}, g^{\prime}(0)\right)$ satisfying $g^{\prime}=\varphi \circ g$. Since $g^{*}\left(\varphi^{*} \mu\right)=g^{* *} \mu=g^{*} \mu$, we see $\varphi^{*} \mu=\mu$.

In particular the Lagrangian lifting $\tilde{f}:\left(\mathbf{R}^{n+k}, 0\right) \rightarrow \mathbf{R}^{2(n+k)}$ of a coisotropic mapgerm $f:\left(\mathbf{R}^{n+k}, 0\right) \rightarrow \mathbf{R}^{2 n}$ with regular reduction is uniquely determined up to symplec- 
tomorphisms preserving the symplectic projection $\pi_{1}: \mathbf{R}^{2(n+k)} \rightarrow \mathbf{R}^{2 n}$. Thus we are able to define the notion of symplectic codimension of $f$ by means of its Lagrangian lifting $\tilde{f}$.

Recall that a Lagrangian submanifold in $\mathbf{R}^{2 n} \times \mathbf{R}^{2 n}=\mathbf{R}^{4 n}(k=n)$ can be regarded as (the graph of) a symplectic relation via both symplectic projections $\pi_{1}: \mathbf{R}^{2 n} \times \mathbf{R}^{2 n} \rightarrow$ $\mathbf{R}^{2 n}$ and $\pi_{2}: \mathbf{R}^{2 n} \times \mathbf{R}^{2 n} \rightarrow \mathbf{R}^{2 n}$ ([19]). Moreover a Lagrangian variety with singularities in $\mathbf{R}^{2 n} \times \mathbf{R}^{2 n}$ has an important role as a singular symplectic relation ([12]).

Also in our case, $0<k<n$, we can consider both projections $\pi_{1}: \mathbf{R}^{2 n} \times \mathbf{R}^{2 k} \rightarrow \mathbf{R}^{2 n}$ and $\pi_{2}: \mathbf{R}^{2 n} \times \mathbf{R}^{2 k} \rightarrow \mathbf{R}^{2 k}$. For a Lagrangian variety $F:\left(\mathbf{R}^{n+k}, 0\right) \rightarrow \mathbf{R}^{2 n} \times \mathbf{R}^{2 k}$, $f=\pi_{1} \circ F$ is, by definition, a coisotropic map-germ, while $g=\pi_{2} \circ F$.

Let $\left(\mathbf{R}^{n+k}, 0\right) \hookrightarrow\left(\mathbf{R}^{2 n}, \omega\right)$ be a coisotropic submanifold-germ. This means that its tangent spaces are coisotropic subspaces of the whole tangent spaces. Thus there exists a unique submersion $g:\left(\mathbf{R}^{n+k}, 0\right) \rightarrow\left(\mathbf{R}^{2 k}, \mu\right)$ onto the symplectic space up to symplectomorphisms such that

$$
\left.\omega\right|_{\mathbf{R}^{n+k}}=\pi^{*} \mu .
$$

This procedure appears as the essential part of a regular symplectic reduction (MarsdenWeinstein reduction). Thus we have generalized it to non-immersive map-germs $f$.

A coisotropic map-germ $f: \mathbf{R}^{n+k} \rightarrow \mathbf{R}^{2 n}$ is a coisotropic immersion (in the ordinary sense) in a neighborhood of a point where $f$ is an immersion and $g$ is a submersion. In fact, at least outside the singular locus of $f$ and $g$, the map-germ $g: \mathbf{R}^{n+k} \rightarrow \mathbf{R}^{2 k}$ defines the symplectic reduction of $f$ on the image $f\left(\mathbf{R}^{n+k}\right) \subset \mathbf{R}^{2 n}$, and fibers of $g$ are pullbacks of characteristic leaves of $f\left(\mathbf{R}^{n+k}\right)$.

The condition that $g$ is a submersion means that the reduction of $f$ has no singularities while $f$ itself may have singularities.

For the generalities on singular symplectic reduction, see [17].

We consider the classification of coisotropic map-germs $f: \mathbf{R}^{n+k} \rightarrow \mathbf{R}^{2 n}$. If $f$ is a coisotropic immersion, then the symplectomorphism class is uniquely determined. Thus we suppose $f$ is not an immersion. Then, together with non-immersive points, $f$ may have self-intersections. Therefore the classification problem of coisotropic map-germs under symplectomorphisms is related, for instance, to the classification problem of pairs of submanifolds in a symplectic space (cf. [14], [15], [2]). In this paper, we give a typical example of coisotropic map-germs, in the case $n=2, k=1$. As the classification problem of glancing hypersurfaces induces interesting results, the classification problem of generic map-germs, say, Whitney umbrellas $\mathbf{R}^{3} \rightarrow \mathbf{R}^{4}$ by symplectomorphisms induces meaningful results, which we are going to show. Note that, in [18], Voronin considered the symplectic classification of the product of a swallowtail and a line in $\mathbf{R}^{4}$. However the symplectic classification of Whitney umbrellas in $\mathbf{R}^{4}$ has never been considered so far.

Consider a parametric hypersurface $f:\left(\mathbf{R}^{3}, 0\right) \rightarrow \mathbf{R}^{4}$ defined by

$$
f(u, v, w)=\left(p_{1}, q_{1}, p_{2}, q_{2}\right)=\left(u v, u^{2}, w, v\right) .
$$

Then $f$ is coisotropic with $g:\left(\mathbf{R}^{3}, 0\right) \rightarrow \mathbf{R}^{2}$,

$$
g(u, v, w)=\left(p_{3}, q_{3}\right)=\left(w-\frac{2}{3} u^{3}, v\right) .
$$

Since $g$ is a submersion, $f$ is coisotropic with regular reduction. The germ $f$ is the so-called 
a Whitney umbrella in 4-space and it has singular locus $\{u=v=0\}$. Moreover $f$ has self-intersection locus $\{v=0\} \subset \mathbf{R}^{3}$ which is mapped as $(u, 0, w) \mapsto\left(u^{2}, 0, w\right)$ by $f$.

It is well known that Whitney umbrella is a generic and stable singularity among smooth mappings $\mathbf{R}^{3} \rightarrow \mathbf{R}^{4}$ : Any smooth mapping $\mathbf{R}^{3} \rightarrow \mathbf{R}^{4}$ can be approximated by a smooth mapping with only Whitney umbrellas as singularities and any small perturbations of a Whitney umbrella are equivalent to the original Whitney umbrella via local diffeomorphisms of $\mathbf{R}^{4}$ and $\mathbf{R}^{3}$. Nevertheless the above germ $f$ is not generic and not stable via symplectic equivalence: In fact, the characteristic foliation is defined by $\left\{v=\right.$ const, $w-\frac{2}{3} u^{3}=$ const $\}$, and so the self-intersection locus is a union of characteristic curves. Such situation is clearly non-generic symplectically.

We remark that the symplectomorphism class of the Lagrange lifting

$$
\tilde{f}=(f, g)=\left(u v, u^{2}, w, v ; w-\frac{2}{3} u^{3}, v\right):\left(\mathbf{R}^{3}, 0\right) \rightarrow \mathbf{R}^{6}
$$

is the 3 -dimensional open Whitney umbrella of type 1.

Recall that the generic singularities of parametric Lagrange varieties $\left(\mathbf{R}^{m}, 0\right) \rightarrow$ $\left(\mathbf{R}^{2 m}, 0\right)$ of corank $\leq 1$ are given by the $m$-dimensional open Whitney umbrella $f_{m, r}$ of type $r(0 \leq 2 r \leq m)$, defined as follows: Define the Whitney umbrella $\left(\mathbf{R}^{m}, 0\right) \rightarrow$ $\left(\mathbf{R}^{m+1}, 0\right)$,

$$
\begin{aligned}
& q_{1}=u^{r+1}+v_{1} u^{r-1}+\cdots+v_{r-1} u, \\
& p_{1}=v_{r} u^{r}+v_{r+1} u^{r-1}+\cdots+v_{2 r-1} u, \\
& q_{2}=v_{1}, \quad \ldots, \quad q_{m}=v_{m-1},
\end{aligned}
$$

parametrized by $\left(u, v_{1}, \ldots, v_{m-1}\right) \in \mathbf{R}^{m}$. Then other components of $f_{m, r}$ are defined by

$$
p_{j}=\int\left(\frac{\partial p_{1}}{\partial v_{j-1}} \frac{\partial q_{1}}{\partial u}-\frac{\partial p_{1}}{\partial u} \frac{\partial q_{1}}{\partial v_{j-1}}\right) d u
$$

$(j=2, \ldots, m)$.

Now consider another example of coisotropic map-germ $f:\left(\mathbf{R}^{3}, 0\right) \rightarrow \mathbf{R}^{4}$ defined by

$$
f(u, v, w)=\left(p_{1}, q_{1}, p_{2}, q_{2}\right)=\left(u v+u^{3}, u^{2}, w, v\right) .
$$

(the $u^{3}$-term added), with the same reduction

$$
g(u, v, w)=\left(p_{3}, q_{3}\right)=\left(w-\frac{2}{3} u^{3}, v\right)
$$

as above. Then the self-intersection locus is in general position with the characteristic foliation (fibers of $g$ ). Actually characteristic curves are transverse to the self-intersection locus outside of singular locus, while, along the singular locus, characteristic curves are simply tangent to the self-intersection locus. Below we show that the latter $f$ is of symplectic codimension zero, and is symplectically stable, while the former's symplectic codimension is infinite.

6. Symplectic codimension of coisotropic varieties. In this section we define the notion of symplectic codimension of coisotropic map-germs with regular reductions.

We define the symplectic codimension of $f:\left(\mathbf{R}^{n+k}, 0\right) \rightarrow\left(\mathbf{R}^{2 n}, 0\right)$ by

$$
\operatorname{sp}-\operatorname{cod}(f)=\operatorname{dim}_{\mathbf{R}} \frac{V C_{f}}{t f\left(V_{n+k}\right)+w f\left(V H_{2 n}\right)} .
$$


The space $V C_{f}$ consists of infinitesimal deformations of $f$ which lift to isotropic deformations of $\tilde{f}$. The subspace $t f\left(V_{n+k}\right)+w f\left(V H_{2 n}\right)$ consists of deformations coming from diffeomorphisms of $\mathbf{R}^{n+k}$ and symplectomorphisms of $\mathbf{R}^{2 n}$. Since $\tilde{f}$ is uniquely determined (Lemma 5.1), the symplectic codimension of $f$ is well-defined.

A detailed formulation goes as follows: Recall that the space of infinitesimal isotropic deformations of $\tilde{f}$ is defined by

$$
V I_{\tilde{f}}:=\left\{v:\left(\mathbf{R}^{n+k}, 0\right) \rightarrow T \mathbf{R}^{2(n+k)} \mid \pi \circ v=\tilde{f}, v^{*}(\dot{\Omega})=0\right\},
$$

as a subspace of the total space

$$
V_{\tilde{f}}:=\left\{v:\left(\mathbf{R}^{n+k}, 0\right) \rightarrow T \mathbf{R}^{2(n+k)} \mid \pi \circ v=\tilde{f}\right\}
$$

of infinitesimal deformations of $\tilde{f}$ (cf. [6]). Here $\pi: T \mathbf{R}^{2(n+k)} \rightarrow \mathbf{R}^{n+k}$ is the bundle projection and $\dot{\Omega}$ is the induced symplectic form on $T \mathbf{R}^{2(n+k)}$ from the standard symplectic form $d \theta$ on $T^{*}\left(\mathbf{R}^{2(n+k)}\right)$ via the isomorphism $T \mathbf{R}^{2(n+k)} \cong T^{*}\left(\mathbf{R}^{2(n+k)}\right)$ induced by the symplectic form $\Omega:=\omega \ominus \mu$ on $\mathbf{R}^{2(n+k)}$. The symplectic form $\dot{\Omega}$ can be regarded also as the natural lifting of $\Omega([7])$.

The differential $\pi_{1 *}: T \mathbf{R}^{2(n+k)} \rightarrow T \mathbf{R}^{2 n}$ of $\pi_{1}: \mathbf{R}^{2(n+k)}=\mathbf{R}^{2 n} \times \mathbf{R}^{2 k} \rightarrow \mathbf{R}^{2 n}$ induces the projection $\Pi: V_{\tilde{f}} \rightarrow V_{f}$, where

$$
V_{f}:=\left\{w:\left(\mathbf{R}^{n+k}, 0\right) \rightarrow T \mathbf{R}^{2 n} \mid \pi^{\prime} \circ w=f\right\},
$$

for the projection $\pi^{\prime}: T \mathbf{R}^{2 n} \rightarrow \mathbf{R}^{2 n}$. Then we set

$$
V C_{f}:=\Pi\left(V I_{\tilde{f}}\right) .
$$

The pushforward $t f: V_{n+k} \rightarrow V_{f}$ from the space of vector fields over $\left(\mathbf{R}^{n+k}, 0\right)$ is defined by $t f(\xi)=f_{*}(\xi),\left(\xi \in V_{n+k}\right)$. Then $t f\left(V_{n+k}\right) \subseteq V C_{f}$. On the other hand the pullback $w f: V_{2 n} \rightarrow V_{f}$ from the space of vector fields over $\left(\mathbf{R}^{2 n}, 0\right)$ is defined by $w f(\eta)=$ $\eta \circ f,\left(\eta \in V_{2 n}\right)$. Among $V_{2 n}$, we denote by $V H_{2 n}$ the space of Hamiltonian vector fields over $\left(\mathbf{R}^{2 n}, 0\right)$. Then we see $w f\left(V H_{2 n}\right) \subseteq V C_{f}$.

Now we show that the symplectic codimension is a diffeomorphism invariant in an appropriate sense:

Proposition 6.1. Let $f, f^{\prime}:\left(\mathbf{R}^{n+k}, 0\right) \rightarrow \mathbf{R}^{2 n}$ be coisotropic map-germs with regular reductions. Suppose $f$ and $f^{\prime}$ are $C^{\infty}$ normalizations ([9]). Then

$$
\operatorname{sp}-\operatorname{cod}(f)=\operatorname{dim}_{\mathbf{R}} \frac{R_{\tilde{f}}}{f^{*} \mathcal{E}_{2 n}+g^{*} \mathcal{E}_{2 n}} .
$$

In particular, if $\left(\tilde{f}, \pi_{1}, \pi_{2}\right)$ and $\left(\tilde{f}^{\prime}, \pi_{1}, \pi_{2}\right)$ are diffeomorphic as divergent diagrams of mappings, then $\operatorname{sp}-\operatorname{cod}(f)=\operatorname{sp}-\operatorname{cod}\left(f^{\prime}\right)$.

Here we set

$$
R_{\tilde{f}}:=\left\{h \in E_{n+k} \mid d h \in E_{n+k} d \tilde{f}\right\} .
$$

Recall that the diffeomorphism type of the Lagrangian lifting $\left(\tilde{f}, \pi_{1}, \pi_{2}\right)$ is uniquely determined by $f$.

Proof of Proposition 6.1. If $f$ and $f^{\prime}$ are right equivalent, then clearly $\operatorname{sp-cod}(f)=$ sp-cod $\left(f^{\prime}\right)$. So we suppose $f^{\prime}=\varphi \circ f$ and $\tilde{f}^{\prime}=\Phi \circ \tilde{f}$ for a diffeomorphism $\Phi:\left(\mathbf{R}^{2(n+k)}, \tilde{f}(0)\right)$ 
$\rightarrow\left(\mathbf{R}^{2(n+k)}, \tilde{f}^{\prime}(0)\right)$ inducing a diffeomorphism $\varphi:\left(\mathbf{R}^{2 n}, f(0)\right) \rightarrow\left(\mathbf{R}^{2 n}, f^{\prime}(0)\right)$ via $\pi_{1}$. We write down the exact sequence:

$$
0 \rightarrow \frac{V C_{f}^{\prime}}{t f\left(V_{n+k}\right)+w f\left(V H_{2 n}^{\prime}\right)} \rightarrow \frac{V C_{f}}{t f\left(V_{n+k}\right)+w f\left(V H_{2 n}\right)} \rightarrow \frac{\widetilde{R}_{f}}{f^{*} E_{2 n}} \rightarrow 0 .
$$

Since $f$ is a $C^{\infty}$ normalization, we see that $w f\left(V H_{2 n}^{\prime}\right) \subseteq V C_{f}^{\prime}$. Thus we have the exact sequence

$$
0 \rightarrow \frac{V C_{f}^{\prime}}{t f\left(V_{n+k}\right)} \rightarrow \frac{V C_{f}}{t f\left(V_{n+k}\right)+w f\left(V H_{2 n}\right)} \rightarrow \frac{\widetilde{R}_{f}}{f^{*} E_{2 n}} \rightarrow 0 .
$$

Since $\operatorname{dim}_{\mathbf{R}} V C_{f}^{\prime} / t f\left(V_{n+k}\right)=\operatorname{dim}_{\mathbf{R}} V C_{f^{\prime}}^{\prime} / t f^{\prime}\left(V_{n+k}\right)$, and moreover $\Phi_{*}$ induces an isomorphism between $\widetilde{R}_{f}=R_{\tilde{f}} / g^{*} E_{2 k}$ and $\widetilde{R}_{f^{\prime}}=R_{\tilde{f}} / g^{\prime *} E_{2 k}$, we see the required invariance.

REMARK 6.1. We have the inequality $\mathrm{sp}-\operatorname{cod}(\tilde{f}) \leq \mathrm{sp}-\operatorname{cod}(f)$. Note that, for a parametric Lagrange variety $\left(\mathbf{R}^{m}, 0\right) \rightarrow\left(\mathbf{R}^{2 m}, 0\right)$ of corank $\leq 1, \operatorname{sp}-\operatorname{cod}(\tilde{f})=0$ is equivalent to the fact that $\tilde{f}$ is symplectomorphic to an open Whitney umbrella.

DeFinition 6.1. We call $f$ infinitesimally symplectically stable if

$$
V C_{f}=t f\left(V_{n+k}\right)+w f\left(V H_{2 n}\right) .
$$

Corollary 6.1. Let $f:\left(\mathbf{R}^{n+k}, 0\right) \rightarrow \mathbf{R}^{2 n}$ be a coisotropic map-germ with regular reduction. Suppose $f$ is a $C^{\infty}$ normalization. Then $f$ is infinitesimally symplectically stable if and only if $R_{\tilde{f}}=f^{*} E_{2 n}+g^{*} E_{2 k}$.

COROLlaRY 6.2. If the Lagrange lifting $\tilde{f}$ is an open Whitney umbrella, then $R_{\tilde{f}}=$ $\tilde{f}^{*} \mathcal{E}_{2(n+k)}$. Therefore

$$
\operatorname{sp-cod}(f)=\operatorname{dim}_{\mathbf{R}} \frac{\tilde{f}^{*} \mathcal{E}_{2(n+k)}}{f^{*} \mathcal{E}_{2 n}+g^{*} \mathcal{E}_{2 n}} .
$$

ExAmple 6.1. Let $f:\left(\mathbf{R}^{3}, 0\right) \rightarrow \mathbf{R}^{4}$ be defined by

$$
f(u, v, w)=\left(p_{1}, q_{1}, p_{2}, q_{2}\right)=\left(u v+u^{3}, u^{2}, w, v\right)
$$

and

$$
g(u, v, w)=\left(p_{3}, q_{3}\right)=\left(w-\frac{2}{3} u^{3}, v\right) .
$$

Then we have $R_{f, g}\left(=R_{f}\right)=f^{*} \mathcal{E}_{4}+g^{*} \mathcal{E}_{2}$. Therefore $f$ is infinitesimally symplectically stable.

Moreover let us consider the coisotropic fibration $H: \mathbf{R}^{4} \rightarrow \mathbf{R}$ defined by $H\left(p_{1}, q_{1}, p_{2}, q_{2}\right)$ $=p_{2}$. Then we have the induced coisotropic bifurcation from $f, F:\left(\mathbf{R}^{2}, 0\right) \rightarrow \mathbf{R}^{2} \times \mathbf{R}$,

$$
F(u, v)=\left(u v+u^{3}, u^{2} ; v\right) .
$$

The bifurcation is actually $A_{1}$-type symplectic bifurcation of planar curves [8].

7. Parametric Lagrangian foliations. Now assume that $f$ is coisotropic and that we can take $g$ of rank $\geq k$. If $f$ is coisotropic with regular reduction, then $f$ satisfies the assumption. Then there exists a Lagrangian projection $\pi: \mathbf{R}^{2 k} \rightarrow \mathbf{R}^{k}$ such that $\pi \circ \tilde{f}$ is a submersion. Then $(\pi \circ \tilde{f})^{-1}(a)$ is a smooth submanifold-germ of $\left(\mathbf{R}^{n+k}, 0\right)$ of 
dimension $n$, and $\left.f\right|_{(\pi \circ \tilde{f})^{-1}(a)}$ is an isotropic map-germ into $\left(\mathbf{R}^{2 n}, \omega\right),\left(a \in\left(\mathbf{R}^{k}, 0\right)\right)$. In local coordinates: $f_{a}^{*} \omega=0, f_{a}(x)=f(x, a)$. Conversely we have:

Proposition 7.1. Let $f_{a}:\left(\mathbf{R}^{n}, 0\right) \rightarrow \mathbf{R}^{2 n}$ be a family of isotropic map-germs ( $a \in$ $\left.\left(\mathbf{R}^{k}, 0\right)\right)$. Then $f:\left(\mathbf{R}^{n+k}, 0\right) \rightarrow \mathbf{R}^{2 n}$ defined by $f(x, a)=f_{a}(x)$ is coisotropic with $g:$ $\left(\mathbf{R}^{n+k}, 0\right) \rightarrow \mathbf{R}^{2 k}$ satisfying $f^{*} \omega=g^{*} \mu$ and $\operatorname{rank}(g) \geq k$.

Proof. By the assumption we see $f^{*} \omega$ is zero when restricted to each $\mathbf{R}^{n} \times a \subset \mathbf{R}^{n+k}$. Therefore $f^{*} \omega=g_{1}(x, a) d a_{1}+\cdots+g_{k}(x, a) d a_{k}$ for some functions $g_{1}, \ldots, g_{k}:\left(\mathbf{R}^{n+k}, 0\right)$

$\rightarrow \mathbf{R}$, where $a_{1}, \ldots, a_{k}$ are coordinate functions on $\left(\mathbf{R}^{k}, 0\right)$ (regarded as functions on $\left(\mathbf{R}^{n+k}, 0\right)$ via the projection $\left.\mathbf{R}^{n+k}=\mathbf{R}^{n} \times \mathbf{R}^{k} \rightarrow \mathbf{R}^{k}\right)$. Set $g=\left(g_{1}, \ldots, g_{k}, a_{1}, \ldots, a_{k}\right)$. Then we have $f^{*} \omega=g^{*} \mu$.

EXAMPLE 7.1. Let us consider the map-germ $f:\left(\mathbf{R}^{3}, 0\right) \rightarrow \mathbf{R}^{4}$ defined by

$$
f(u, v, w)=\left(p_{1}, p_{2}, q_{1}, q_{2}\right)=\left(u w^{2}, v w^{2}, u, v\right) .
$$

Then

$$
f^{*} \omega=2 v w d w \wedge d u+2 v w d w \wedge d v=g^{*} \mu,
$$

with the singular reduction

$$
g=\left(p_{3}, q_{3}\right)=\left(\frac{1}{2}\left(u^{2}+v^{2}+w^{2}\right), \frac{1}{2}\left(u^{2}+v^{2}-w^{2}\right)\right),
$$

of rank zero. Therefore $f$ is coisotropic with singular reduction. In this case $f$ is foliated by singular Lagrangian varieties which are not necessarily parametrized by smooth manifolds.

\section{References}

[1] V. I. Arnold, Lagrangian manifold singularities, asymptotic rays and the open swallowtail, Funct. Anal. Appl. 15 (1981), 235-246.

[2] V. I. Arnold, Singularities of Caustics and Wave Fronts, Kluwer Academic Publishers, Dordrecht, 1990.

[3] V. I. Arnold and A. B. Givental, Symplectic geometry, in: Dynamical Systems, IV, Encyclopaedia Math. Sci. 4, Springer, Berlin, 2001, 1-138.

[4] W. Domitrz, S. Janeczko and M. Zhitomirskii, Symplectic singularities of varieties: The method of algebraic restrictions, J. Reine Angew. Math. 618 (2008), 197-235.

[5] A. B. Givental', Singular Lagrangian manifolds and their Lagrangian mappings, Itogi Nauki Tekh., Ser. Sovrem. Prob. Mat. 33, VINITI, 1988, 55-112; English transl.: J. Soviet Math. 52 (1990), 3246-3278.

[6] G. Ishikawa, Symplectic and Lagrange stabilities of open Whitney umbrellas, Invent. Math. 126 (1996), 215-234.

[7] G. Ishikawa, Infinitesimal deformations and stabilities of singular Legendre submanifolds, Asian J. Math. 9 (2005), 133-166.

[8] G. Ishikawa and S. Janeczko, Symplectic bifurcations of plane curves and isotropic liftings, Quarterly Journal of Mathematics 54 (2003), 73-102.

[9] G. Ishikawa and S. Janeczko, Symplectic singularities of isotropic mappings, in: Geometric Singularity Theory, H. Hironaka et al. (eds.), Banach Center Publications 65, 2004, 85-106. 
[10] G. Ishikawa and S. Janeczko, The complex symplectic moduli spaces of parametric plane curve singularities, preprint, 2006.

[11] S. Janeczko, Coisotropic varieties and their generating families, Ann. Inst. Henri Poincaré 56 (1992), 429-441.

[12] S. Janeczko, Lagrangian submanifolds in product symplectic spaces, J. Math. Phys. 41 (2000), 5642-5655.

[13] E. Lerman, R. Montgomery and R. Sjamaar, Examples of singular reduction, in: Symplectic Geometry, London Math. Soc. Lecture Note Ser. 192, Cambridge Univ. Press, Cambridge, 1993, 127-155.

[14] R. Melrose, Equivalence of glancing hypersurfaces, Invent. Math. 37 (1976), 165-191.

[15] R. Melrose, Equivalence of glancing hypersurfaces II, Math. Ann. 255 (1981), 159-198.

[16] M. Mikosz, On classification of the linear Lagrangian and isotropic subspaces, Demonstratio Math. 30 (1997), 437-450.

[17] R. Sjamaar and E. Lerman, Stratified symplectic spaces and reduction, Ann. of Math. 134 (1991), 375-422.

[18] S. M. Voronin, The Darboux-Whitney theorem and related questions, in: Nonlinear Stokes Phenomena, S. Il'yashenko (ed.), Advances in Soviet Mathematics 14 (1993), 139-233.

[19] A. Weinstein, Symplectic geometry, Bull. Amer. Math. Soc. 5 (1981), 1-13. 